\title{
An ECG-Based System for Respiratory Rate Estimation Tested on a Wearable Armband during Daily Life
}

\author{
Jesus Lazaro ${ }^{1,2,3}$, Natasa Reljin ${ }^{3}$, Raquel Bailón ${ }^{1,2}$, Eduardo Gil ${ }^{1,2}$, \\ Yeonsik $\mathrm{Noh}^{4}$, Pablo Laguna ${ }^{1,2}$, Ki H Chon ${ }^{3}$ \\ ${ }^{1}$ BSICoS Group, Aragón Institute for Engineering Research (I3A), IIS Aragón, \\ University of Zaragoza, Zaragoza, Spain \\ ${ }^{2}$ CIBER in Bioengineering, Biomaterials and Nanomedicine (CIBER-BBN), Madrid, Spain \\ ${ }^{3}$ Department of Biomedical Engineering, University of Connecticut, Storrs, CT, USA \\ ${ }^{4}$ College of Nursing and Department of Electrical and Computer Engineering, \\ University of Massachusetts, Armherst, MA, USA
}

\begin{abstract}
A pilot study on breathing rate (BR) estimation during daily life by using a wearable armband is presented. This wearable armband provides three electrocardiogram (ECG) channels, and BR was estimated from them by using ECG derived respiration (EDR) techniques based on respiration-related QRS morphology modulations: QRS slopes and $R$-wave angle. Five healthy volunteers wore the armband during 24 hours, with the only instruction not to exercise. In addition, reference ECG signals were simultaneously recorded by a market-available 3-channel Holter monitor. The percentage of armband's accurate $B R$ estimations (differing less than 5\% from the Holter estimation) with respect to the total number of Holter's estimations was computed $\left(P_{1}\right)$. In addition, the percentage of accurate armband's BR estimations with respect to the total number of armband's estimations was also computed $\left(P_{2}\right) . P_{1}$ ranged from $26.59 \%$ to $73.00 \%$ during non-bed time, and from $63.05 \%$ to $88.73 \%$ during bed time. $P_{2}$ ranged from $60.89 \%$ to $94.57 \%$ during non-bed time, and from $81.65 \%$ to $97.38 \%$ during bed time. These results are promising and suggest that the armband may be useful for $B R$ monitoring in some applications. However, an artifact detector specifically focused on detecting those segments which are usable for BR detection needs to be developed.
\end{abstract}

\section{Introduction}

A wearable armband for long-term electrocardiogram (ECG) monitoring is being developed in our lab at University of Connecticut [1]. The ECG monitoring allows, among other things, to measure some cardiac vital signs such as heart rate and its variability, and to estimate respi- ratory information such as breathing rate (BR) through the so-called ECG-derived respiration (EDR) techniques [2]. Heart rate and BR monitoring by using a wearable device would have a wide range of applications, including sleep studies, epileptic seizure detection, stress level assessment, and monitoring chronic respiratory patients [3].

The armband device is designed to be worn on the left upper-arm. It records 3 ECG channels by using 3 pairs of dry electrodes which do not cause skin irritation when used for long periods of time, in difference to the conventional wet (hydrogel) electrodes [4]. The armband has previously been evaluated for heart rate monitoring during daily life, obtaining accurate heart rate during $75.25 \%$ (in median) of the non-bed time, and during $98.49 \%$ (in median) of the bed time [1]. Furthermore, the armband has previously been evaluated also for estimating BR during lab-controlled restricted-movement conditions, showing a relative error not higher than $1.40 \%$ in median (intersubject median of intra-subject median) and $3.06 \%$ in IQR (inter-subject median of intra-subject IQR) [3]. However, the armband has never been evaluated for BR estimation during daily life. Artifacts are certainly expected during daily life, mainly due to the electromyogram contribution from the left upper-arm [1]. Therefore, the BR estimation is expected not to always be possible during daily life, and, when possible, is expected not to be as accurate as it is during lab-controlled restricted-movement conditions.

In this paper, a pilot study on estimation of BR with the armband during daily life is presented. An EDR technique based on QRS slopes and R-wave angle was used together with a fusion method that combines the information in the different EDR signals based on the shape of their spectra. Furthermore, the output of this fusion technique becomes a "non-estimable rate" in those cases in which none of the spectra has an appropriate shape. A conventional Holter 
monitor available in the market was used as reference. The coverage of the armband in BR estimation terms is analyzed, by computing the percentage of time that the armband provides an accurate BR estimation with respect to the total time that the reference offers a BR estimation. Moreover, the reliability of the armband's BR estimates is also analyzed, by computing the percentage of the time that the armband offers an accurate BR estimate with respect to the time when it is offering an estimate.

\section{Materials and methods}

\subsection{Data and signal preprocessing}

Five healthy volunteers worn the wearable armband during 24 hours. These subjects were instructed to do their normal lives with the exception of not exercising. For reference purposes, three ECG channels were simultaneously recorded by a conventional Holter available in the market: Rozinn RZ 153+ (Glendale, NY, USA). The experimental protocol was approved by the Institutional Review Board at the University of Connecticut (Protocol H16-107).

A band-pass filter with cut-off frequencies $3 \mathrm{~Hz}$ and 25 $\mathrm{Hz}$, for attenuating the electromyogram (EMG) component [1]. An additional ECG channel was computed as the first principal component obtained from a principal component analysis, as in [3]. An example of the preprocessed ECG channels can be observed in Fig. 1.

Artifacts in the armband-ECG channels were detected by the SVM-based artifact detector presented in [1], which was specifically designed for the armband-ECG signals. R-wave peaks $\left(n_{\mathrm{R}_{i}}\right)$ were detected by an automatic detector based on variable frequency complex demodulation [5]. Subsequently, Q and S points were detected as the instants where ECG shows minimum amplitude within a window of $40 \mathrm{~ms}$ before and after the $\mathrm{R}$ point, respectively.

\subsection{ECG-derived respiration}

An EDR algorithm based on QRS slopes and R-wave angle was used. This algorithm offered accurate estimations of respiratory rate from the armband-ECG signals during restricted-movement conditions in [3]. First, the points of maximum variation between $\mathrm{Q}$ and $\mathrm{R}$, and between $\mathrm{R}$ and $\mathrm{S}$ were detected. Then, a straight line was fitted to the ECG signal within $8 \mathrm{~ms}$ around each one of the two maximum variation points. The slopes of these lines $\mathcal{I}_{\mathrm{Us}_{i}}$ and $\mathcal{I}_{\mathrm{DS}_{i}}$, respectively) are estimations of the $\mathrm{QRS}$ slopes, and they are used for computing two QRS morphology parameters:

$$
\begin{aligned}
\mathcal{I}_{\mathrm{SR}_{i}} & =\mathcal{I}_{\mathrm{US}_{i}}-\mathcal{I}_{\mathrm{DS}_{i}} \\
\Phi_{i} & =\frac{\mathcal{I}_{\mathrm{US}_{i}}-\mathcal{I}_{\mathrm{DS}_{i}}}{0.4\left(6.25+\mathcal{I}_{\mathrm{US}_{i}} \mathcal{I}_{\mathrm{DS}_{i}}\right)}
\end{aligned}
$$

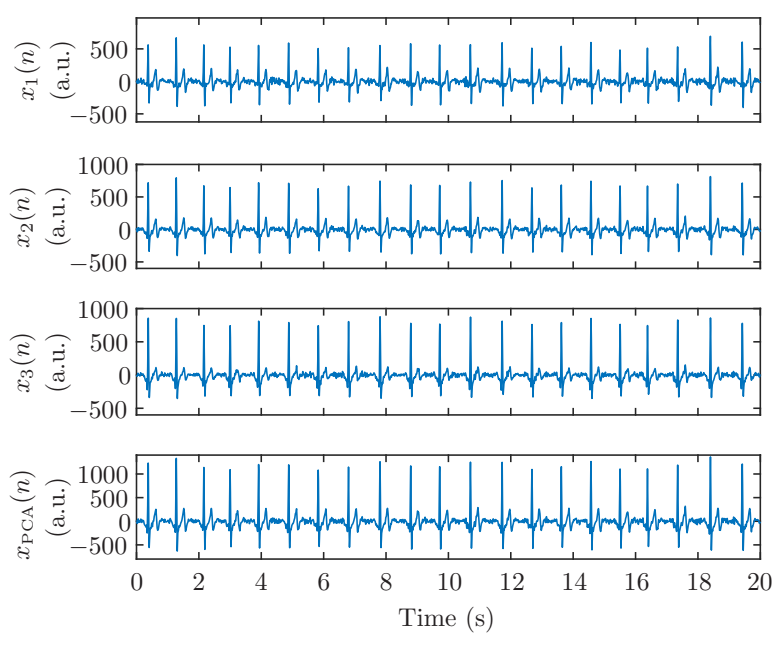

Figure 1. 20 seconds of armband ECG channels.

where $\mathcal{I}_{\mathrm{SR}_{i}}$ and $\Phi_{i}$ are the QRS slope range (SR) and the $\mathrm{R}$-wave angle of the ith QRS complex, respectively. Concatenation of these morphological parameters from the different QRS complexes of one ECG channel leads to an unevenly sampled EDR signal:

$$
d_{\{\mathrm{SR}, \Phi\}}^{u}=\sum_{i}\left\{\mathcal{I}_{\mathrm{SR}_{i}}, \Phi_{i}\right\} \delta\left(n-n_{\mathrm{R}_{i}}\right)
$$

These EDR signals were further interpolated by cubic splines in order to obtain evenly-sampled EDR signals $d_{\{\mathrm{SR}, \Phi\}}$ with a sampling rate of $4 \mathrm{~Hz}$.

\subsection{Respiratory rate estimation}

Estimation of BR was performed by using a timefrequency algorithm that can take one or more EDR signals as inputs [3]. First, a power spectrum density (PSD) is computed from the kth segment of $42 \mathrm{~s}$ from the jth EDR signal used as input $\left(S_{j}(k, f)\right)$. This PSD is computed by using the Welch periodogram, using a length for the windows of $12 \mathrm{~s}$, and shifting with an overlap of $50 \%$. A PSD is obtained each $5 \mathrm{~s}$. The concatenation of successive $S_{j}(k, f)$ leads to a time-frequency PSD of the $j$ th EDR signal used as input.

Then, a new time-frequency map, $\bar{S}(k, f)$, was computed by combining all $S_{j}(k, f)$ available from all EDR signals ( 2 per ECG channel) within the surrounding $25 \mathrm{~s}$. The combination consists of a peak-conditioned average, which is an average of those $S_{j}(k, f)$ that fulfill two criterions on how their power is concentrated or not around a peak. Thus, only those $S_{j}(k, f)$ that have a "peaked" shape take part in the average $\bar{S}(k, f)$. If none of the $S_{j}(k, f)$ within the surrounding 25 s of $\bar{S}(k, f)$ fulfil the "peaked"shape criterions, then the respiratory rate at the instant $\mathrm{k}$ is not estimated. An example of $\bar{S}(k, f)$ obtained from the armband during one hour can be observed in Fig. 2. In 
(a) Armband

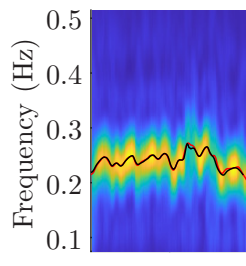

500
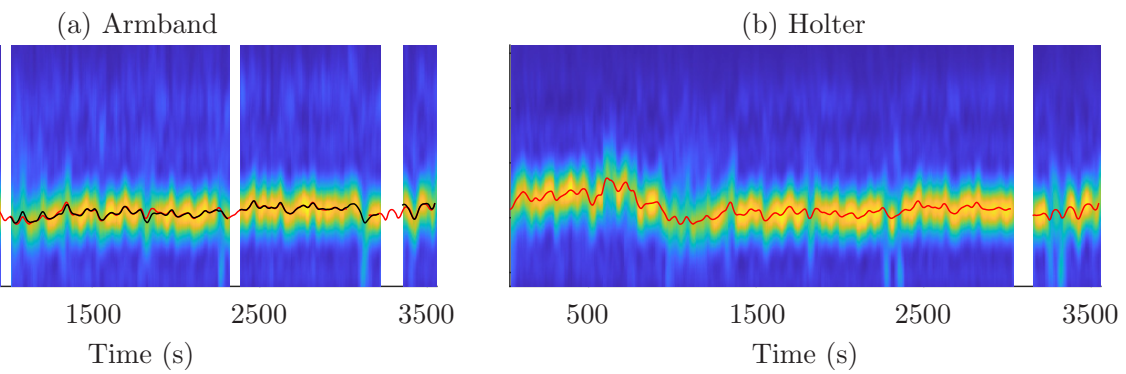

Figure 2. (a) Example of one hour of $\bar{S}(k, f)$ obtained from the armband. The solid black line represents the estimated respiratory rate, and the solid red line represents the reference (estimated from the Holter) respiratory rate. (b) $\bar{S}(k, f)$ obtained from the Holter for the same subject during the same hour. The solid red line represents the reference respiratory rate. The white gaps represent those periods when the BR was not estimated.

addition, those $\bar{S}(k, f)$ obtained from the Holter for the same subject during the same hour are also shown. Further details of the algorithm can be found in [6].

\subsection{Performance measures}

Relative error of the respiratory rate estimates from the armband with respect to the respiratory rate estimates from the Holter was computed as:

$$
e(k)=\frac{\hat{f}_{\mathrm{A}}(k)-\hat{f}_{\mathrm{H}}(k)}{\hat{f}_{\mathrm{H}}(k)} \times 100,
$$

where $\hat{f}_{\mathrm{A}}(k)$ and $\hat{f}_{\mathrm{H}}(k)$ are the respiratory rate estimates from the armband and the Holter, respectively.

\section{Results}

Two ratios were computed: the coverage ratio and the reliability ratio. The coverage ratio was computed as the percentage of armband's accurate BR estimations (differing less than 5\% from the Holter estimation) with respect to the total number of Holter's estimations $\left(P_{1}\right)$. The reliability ratio was computed as the percentage of accurate armband's BR estimations with respect to the total number of armband's estimations $\left(P_{2}\right)$. Table 1 shows the obtained ratios $P_{1}$ and $P_{2}$ for the 5 subjects, separately for non-bed time and the bed time.

Table 1. Obtained $P_{1}$ and $P_{2}$ for the 5 subjects (non-bed time / bed time).

\begin{tabular}{lcc}
\hline \hline Subject & $P_{1}(\%)$ & $P_{2}(\%)$ \\
\hline Subject 1 & $26.59 / 78.13$ & $60.89 / 84.42$ \\
Subject 2 & $34.01 / 63.05$ & $75.31 / 93.35$ \\
Subject 3 & $50.08 / 70.12$ & $79.30 / 81.65$ \\
Subject 4 & $49.11 / 76.39$ & $87.44 / 83.29$ \\
Subject 5 & $73.00 / 88.73$ & $94.57 / 97.38$ \\
\hline \hline
\end{tabular}

\section{Discussion}

A pilot study on BR estimation during daily life by using a wearable armband has been presented. This wearable armband provides three ECG channels, and BR was estimated from them by using EDR techniques. The armband was previously reported to successfully monitor BR during lab-controlled restricted-movement conditions [3]. This paper describes the first attempt to BR monitoring using the armband during daily life.

Five healthy volunteers wore the armband during 24 hours, with the only instruction of not exercising. In addition, reference ECG signals were simultaneously recorded by a market-available 3-channel Holter monitor.

The chosen EDR technique exploits respiration-related variations in QRS slopes and R-wave angle. This choice was made because these techniques were shown to be the most robust techniques when tested in different scenarios [7], including highly non-stationary and noisy environments [6]. Furthermore, as these features are based exclusively on QRS morphology, they are applicable to all those situations where the QRS has a conventional morphology, including some arrhythmias such as atrial fibrillation [8].

Artifacts are certainly expected in the armband ECG signals during daily life monitoring [1]. Thus, it was assumed that the BR estimation is not going to be always accurate. The identification of those segments where the BR estimation is accurate remains very interesting for a final application. The methods used in this work contemplate two ways to discard data and so, to offer "non-estimable rate" as output. On one hand, those ECG segments where artifacts were found according to the SVM-based artifact detector presented in [1] were discarded. Thus, in those periods of time when this issue happened with all the 4 available ECG channels, an output of "non-estimable rate" was given. On the other hand, the BR estimation technique described in Section 2.3 discards those EDR signals whose PSD has not a peaky shape. Thus, in those periods of time when none of the available EDR showed a peaky PSD, an output of 
"non-estimable rate" was given.

In the absence of a reference respiratory signal in the data set, the BR estimated from the Holter device was used as reference. This issue remains a limitation in this study since, although the applied EDR methods have shown good performance when applied with conventional ECG setup in different scenarios [7], to the best of our knowledge, they have never been evaluated during daily life.

The armband-based BR estimation was considered accurate if it differs less than $5 \%$ from the reference (Holterbased BR estimation). Two ratios were computed: the percentage of armband's accurate BR estimations with respect to the total number of Holter's estimations $\left(P_{1}\right)$; and the percentage of accurate armband's BR estimations with respect to the total number of armband's estimations $\left(P_{2}\right)$. The first ratio, $P_{1}$, is aimed to quantify the coverage of the armband on BR estimation, i.e., the percentage of the time when the armband is able to offer an accurate BR estimate. The second ratio, $P_{2}$, is aimed to quantify the reliability of the armband's BR estimates, i.e., the percentage of armband's BR estimates that are accurate.

As shown in Table 1, $P_{1}$ ranged from $25.59 \%$ to $73.00 \%$ during the non-bed time, while it ranged from $63.05 \%$ to $88.73 \%$ during the bed time, being higher than during the non-bed time for all the subjects. This behavior was expected, because much less movement is expected during the bed time than during the non-bed time. These results are promising and suggest that the armband has some potential as BR monitor in several applications where a continuous BR estimation is not strictly needed, such as a regular stress level measure, a daily sleep quality quantification, or a regular monitoring of chronic respiratory patients. It is worthy to note that our data was recorded from healthy subjects, who probably have a more active life than a chronic respiratory patient.

However, $P_{2}$ ranged from $60.89 \%$ to $94.57 \%$ during the non-bed time, and from $81.65 \%$ to $97.38 \%$ during the bed time. This suggest that the detection of those segments that are not offering an accurate BR estimation has big room for improvement. A possible reason is that the SVMbased artifact detector was designed to detect those segments from which we can obtain an accurate heart rate estimation [1], i.e., those segments where QRS complexes can be detected. However, QRS-morphology-based BR estimation is more challenging than heart rate estimation, as it exploits the QRS morphology and not only the QRS presence. Therefore, the artifact detector should be retrained for this purpose, focusing on detecting those segments which are usable for BR detection.

\section{Conclusions}

Obtained results in this pilot study are promising and suggest that the armband may be useful for BR monitoring in some applications. However, an artifact detector specifically focused on detecting those segments which are usable for BR detection needs to be developed.

\section{Acknowledgments}

This project has received funding from the European Union's Framework Programme for Research and Innovation Horizon 2020 (2014-2020) under the Marie Skłodowska-Curie Grant Agreement No. 745755. This work was supported also by Gobierno de Aragón (Reference Group BSICoS T39-20R) cofunded by FEDER20142020 "Building Europe from Aragón", Spanish project PID2019-104881RB-I00 and by CIBER-BBNthrough Instituto de Salud Carlos III. This work was also supported by NSF SBIR Phase I (\#1746589) and R43 HL135961.

\section{References}

[1] Lazaro $\mathbf{J}$ et al. Wearable armband device for daily life electrocardiogram monitoring. IEEE Transactions on Biomedical Engineering 2020; early access, DOI: 10.1109/TBME.2020.2987759.

[2] Charlton P et al. An assessment of algorithms to estimate respiratory rate from the electrocardiogram and photoplethysmogram. Physiological Measurement 2016;37(4):610-626, DOI: $10.1088 / 0967-3334 / 37 / 4 / 610$.

[3] Lazaro $\mathbf{J}$ et al. Electrocardiogram derived respiratory rate using a wearable armband. IEEE Transactions on Biomedical Engineering 2020; early access, DOI: 10.1109/TBME.2020.3004730.

[4] Reyes B et al. Novel electrodes for underwater ECG monitoring. IEEE Transactions on Biomedical Engineering 2014; 61(6):1863-1876, DOI: 10.1109/TBME.2014.2309293.

[5] S Bashar et al. VERB: VFCDM-based electrocardiogram reconstruction and beat detection algorithm. IEEE Access 2019;7:13856-13866, DOI: 10.1109/ACCESS.2019.2894092.

[6] Lazaro J et al. Electrocardiogram derived respiratory rate from QRS slopes and R-wave angle. Annals of Biomedical Engineering 2014;42(10):2072-2083, DOI: 10.1007/s 10439-014-1073-x.

[7] Varon $\mathrm{C}$ et al. A comparative study of ECG-derived respiration in ambulatory monitoring using the single-lead ECG. Scientific Reports 2020;10:5704, DOI: 10.1038/s41598020-62624-5.

[8] Kontaxis $\mathrm{S}$ et al. ECG-derived respiratory rate in atrial fibrillation. IEEE Transactions on Biomedical Engineering 2020; 10:905-914, DOI: 10.1109/TBME.2019.2923587.

Address for correspondence:

Jesus Lazaro

Dep. Ingeniería Electrónica y Comunicaciones. Universidad de Zaragoza, C/ María de Luna 1, 50018 Zaragoza, Spain jlazarop@unizar.es 